\title{
JENSEN TYPE INEQUALITIES FOR $Q$-CLASS FUNCTIONS
}

\author{
MOHAMMAD SAL MOSLEHIAN ${ }^{\bowtie}$ and MOHSEN KIAN
}

(Received 2 June 2011)

\begin{abstract}
Some inequalities of Jensen type for $Q$-class functions are proved. More precisely, a refinement of the inequality $f\left((1 / P) \sum_{i=1}^{n} p_{i} x_{i}\right) \leq P \sum_{i=1}^{n}\left(f\left(x_{i}\right) / p_{i}\right)$ is given in which $p_{1}, \ldots, p_{n}$ are positive numbers, $P=\sum_{i=1}^{n} p_{i}$ and $f$ is a $Q$-class function. The notion of the jointly $Q$-class function is introduced and some Jensen type inequalities for these functions are proved. Some Ostrowski and Hermite-Hadamard type inequalities related to $Q$-class functions are presented as well.
\end{abstract}

2010 Mathematics subject classification: primary 26D15; secondary 26A51, 47J99.

Keywords and phrases: $Q$-class function, Jensen's inequality, jointly $Q$-class, Ostrowski type inequality, convex function, Hermite-Hadamard type inequality.

\section{Introduction and preliminaries}

Assume that $p_{1}, \ldots, p_{n}$ are positive numbers and $P=\sum_{i=1}^{n} p_{i}$. If $f: J \rightarrow \mathbb{R}$ is a convex function and $x_{i} \in J(i=1, \ldots, n)$, then the following so-called Jensen inequality holds:

$$
f\left(\frac{1}{P} \sum_{i=1}^{n} p_{i} x_{i}\right) \leq \frac{1}{P} \sum_{i=1}^{n} p_{i} f\left(x_{i}\right) .
$$

A real-valued function $f$ on an interval $J$ is said to be of $Q$-class or to be a $Q$-class function if

$$
f(\lambda x+(1-\lambda) y) \leq \frac{f(x)}{\lambda}+\frac{f(y)}{1-\lambda}
$$

for all $x, y \in J$ and all $\lambda \in(0,1)$. This notion was first introduced by Godunova and Levin [12]. It is easy to see that this class of functions contains all nonnegative monotone increasing functions and nonnegative convex functions. Also, all such functions are nonnegative [11]. Many other properties of such functions can be found in $[8,9,17,18]$. Mitrinović and Pečarić [15] proved the following inequality similar to (1.1) for $Q$-class functions:

$$
f\left(\frac{1}{P} \sum_{i=1}^{n} p_{i} x_{i}\right) \leq P \sum_{i=1}^{n} \frac{f\left(x_{i}\right)}{p_{i}} .
$$

This research was supported by a grant from Ferdowsi University of Mashhad (No. MP90210MOS).

(C) 2011 Australian Mathematical Publishing Association Inc. 0004-9727/2011 \$16.00 
Dragomir and Pearce [8] obtained some Jensen type inequalities for $Q$-class functions. Some new properties of $Q$-class functions are proved in [17, 18].

Recall that a function $f:[a, b] \times[c, d] \rightarrow \mathbb{R}$ is said to be jointly convex if

$$
f\left(\lambda(x, y)+(1-\lambda)\left(x^{\prime}, y^{\prime}\right)\right) \leq \lambda f(x, y)+(1-\lambda) f\left(x^{\prime}, y^{\prime}\right)
$$

for all $x, x^{\prime} \in[a, b], y, y^{\prime} \in[c, d]$ and $\lambda \in[0,1]$. Klaričić Bakula and Pečarić [14] proved some inequalities related to jointly convex functions.

If $f: I \subseteq[0, \infty) \rightarrow \mathbb{R}$ is differentiable on $I^{\circ}$, the interior of $I, a, b \in I, a<b, f$ is integrable on $[a, b]$ and $\left|f^{\prime}(x)\right| \leq M$, then

$$
\left|f(x)-\frac{1}{b-a} \int_{a}^{b} f(t) d t\right| \leq \frac{M}{b-a}\left(\frac{(b-x)^{2}+(x-a)^{2}}{2}\right) .
$$

This is known as the Ostrowski inequality.

If $f: I \subseteq[0, \infty) \rightarrow \mathbb{R}$ is a convex function and $a, b \in I, a<b$, then the following socalled Hermite-Hadamard inequality holds:

$$
f\left(\frac{a+b}{2}\right) \leq \frac{1}{b-a} \int_{a}^{b} f(x) d x \leq \frac{f(a)+f(b)}{2} .
$$

Dragomir et al. [9] obtained a Hermite-Hadamard type inequality for $Q$-class functions. Recently some authors have established some results concerned with the Ostrowski inequality and the Hermite-Hadamard inequality. In [3], some Ostrowski type inequalities for functions, whose derivatives are $s$-convex in the second sense, are proved. Dragomir and Agarwal [7] proved some Hermite-Hadamard type inequalities for differentiable convex functions. A series of other results can be found in $[1,2,4-$ $6,10,13]$.

In Section 2 we give a refinement of (1.2) and state some of its applications. In Section 3 we introduce the notion of a jointly $Q$-class function and investigate some of its properties. We also present some inequalities related to the Jensen inequality for such functions. In the last section, we prove some Ostrowski and Hermite-Hadamard type inequalities for functions whose first or second derivatives are $Q$-class.

\section{Refinement of the Jensen inequality}

In this section we aim to establish some Jensen type inequalities. If $p_{1}>0$ and $p_{i}<0(i=2, \ldots, n)$ are such that $P=\sum_{i=1}^{n} p_{i}>0$, then

$$
f\left(\frac{1}{P} \sum_{i=1}^{n} p_{i} x_{i}\right) \geq P \sum_{i=1}^{n} \frac{f\left(x_{i}\right)}{p_{i}} .
$$

To see this, set $p_{1}=P, x_{1}=(1 / P) \sum_{i=1}^{n} p_{i} x_{i}$ and $p_{i}=-p_{i}(i=2, \ldots, n)$ in (1.2). Now let $X$ be a real vector space and $f: X \rightarrow \mathbb{R}$ be a $Q$-class function. Let $p_{1}, \ldots, p_{n}$ be positive numbers such that $\sum_{i=1}^{n} p_{i}=1$. Let $J$ be a nonempty proper subset 
of $\{1, \ldots, n\}$. Assume that $\bar{J}=\{1, \ldots, n\}-J, P_{J}=\sum_{i \in J} p_{j}$ and $\bar{P}_{J}=P_{\bar{J}}=1-P_{J}$. For $x_{i} \in X(i=1, \ldots, n)$ we put $x=\left(x_{1}, \ldots, x_{n}\right)$. Set

$$
\Omega(f, J, P, x):=\frac{1}{P_{J}} f\left(\frac{1}{P_{J}} \sum_{i \in J} p_{i} x_{i}\right)+\frac{1}{\bar{P}_{J}} f\left(\frac{1}{\bar{P}_{J}} \sum_{i \in J} p_{i} x_{i}\right) .
$$

We obtain the following refinement of (1.2).

THEOREM 2.1. With the notation as above:

$$
\begin{gathered}
f\left(\sum_{i=1}^{n} p_{i} x_{i}\right) \leq \Omega(f, J, P, x) \leq \sum_{i=1}^{n} \frac{f\left(x_{i}\right)}{p_{i}} \\
\sum_{i=1}^{n} \frac{f\left(x_{i}\right)}{p_{i}}-f\left(\sum_{i=1}^{n} p_{i} x_{i}\right) \geq \sum_{i \in J} \frac{f\left(x_{i}\right)}{p_{i}}-\frac{1}{P_{J}} f\left(\frac{1}{P_{J}} \sum_{i \in J} p_{i} x_{i}\right) \geq 0 .
\end{gathered}
$$

In particular,

$$
\begin{aligned}
\sum_{i=1}^{n} \frac{f\left(x_{i}\right)}{p_{i}}-f\left(\sum_{i=1}^{n} p_{i} x_{i}\right) & \geq \max _{1 \leq k, \ell \leq n}\left\{\frac{f\left(x_{k}\right)}{p_{k}}+\frac{f\left(x_{\ell}\right)}{p_{\ell}}-\frac{1}{p_{k}+p_{\ell}} f\left(\frac{p_{k} x_{k}+p_{\ell} x_{\ell}}{p_{k}+p_{\ell}}\right)\right\} \\
& \geq 0 .
\end{aligned}
$$

Proof. (i) It follows from (1.2) that

$$
\begin{aligned}
\Omega(f, J, P, x) & =\frac{1}{P_{J}} f\left(\frac{1}{P_{J}} \sum_{i \in J} p_{i} x_{i}\right)+\frac{1}{\bar{P}_{J}} f\left(\frac{1}{\bar{P}_{J}} \sum_{i \in \bar{J}} p_{i} x_{i}\right) \\
& \leq \frac{1}{P_{J}}\left(P_{J} \sum_{i \in J} \frac{f\left(x_{i}\right)}{p_{i}}\right)+\frac{1}{\bar{P}_{J}}\left(\bar{P}_{J} \sum_{i \in \bar{J}} \frac{f\left(x_{i}\right)}{p_{i}}\right) \\
& =\sum_{i=1}^{n} \frac{f\left(x_{i}\right)}{p_{i}},
\end{aligned}
$$

which is the second inequality of (2.1). To get the first inequality of (2.1), note that $P_{J}+P_{\bar{J}}=1$. We have

$$
\begin{aligned}
\Omega(f, J, P, x) & =\frac{1}{P_{J}} f\left(\frac{1}{P_{J}} \sum_{i \in J} p_{i} x_{i}\right)+\frac{1}{\bar{P}_{J}} f\left(\frac{1}{\bar{P}_{J}} \sum_{i \in \bar{J}} p_{i} x_{i}\right) \\
& \geq f\left(P_{J}\left(\frac{1}{P_{J}} \sum_{i \in J} p_{i} x_{i}\right)+\bar{P}_{J}\left(\frac{1}{\bar{P}_{J}} \sum_{i \in J} p_{i} x_{i}\right)\right) \quad \text { (since } f \text { is } Q \text {-class) } \\
& =f\left(\sum_{i \in J} p_{i} x_{i}+\sum_{i \in \bar{J}} p_{i} x_{i}\right) \\
& =f\left(\sum_{i=1}^{n} p_{i} x_{i}\right) .
\end{aligned}
$$


(ii) By (1.2), the second inequality of (2.2) holds. Also

$$
\begin{aligned}
f\left(\sum_{i=1}^{n} p_{i} x_{i}\right) & =f\left(\sum_{i \in \bar{J}} p_{i} x_{i}+P_{J}\left(\frac{1}{P_{J}} \sum_{i \in J} p_{i} x_{i}\right)\right) \\
& \leq \sum_{i \in \bar{J}} \frac{f\left(x_{i}\right)}{p_{i}}+\frac{1}{P_{J}} f\left(\frac{1}{P_{J}} \sum_{i \in J} p_{i} x_{i}\right)
\end{aligned}
$$

since $\sum_{i \in \bar{J}} p_{i}+P_{J}=1$ and $f$ is $Q$-class. Therefore

$$
\begin{aligned}
\sum_{i=1}^{n} \frac{f\left(x_{i}\right)}{p_{i}}-f\left(\sum_{i=1}^{n} p_{i} x_{i}\right) & \geq \sum_{i=1}^{n} \frac{f\left(x_{i}\right)}{p_{i}}-\sum_{i \in \bar{J}} \frac{f\left(x_{i}\right)}{p_{i}}-\frac{1}{P_{J}} f\left(\frac{1}{P_{J}} \sum_{i \in J} p_{i} x_{i}\right) \\
& =\sum_{i \in J} \frac{f\left(x_{i}\right)}{p_{i}}-\frac{1}{P_{J}} f\left(\frac{1}{P_{J}} \sum_{i \in J} p_{i} x_{i}\right) \geq 0 .
\end{aligned}
$$

Putting $J=\{k, \ell\}$ for some $k, \ell \in\{1, \ldots, n\}$ we obtain (2.3).

Let $f, X$ and $P$ be as above. If $J=\{k\}$ for some $k \in\{1, \ldots, n\}$ we obtain the following consequence.

COROLlary 2.2. Let $f$ be a $Q$-class function on a real vector space $X$ and suppose that $x_{1}, \ldots, x_{n} \in X$. If $p_{1}, \ldots, p_{n}$ are positive numbers such that $\sum_{i=1}^{n} p_{i}=1$, then

$$
\begin{aligned}
f\left(\sum_{i=1}^{n} p_{i} x_{i}\right) & \leq \min _{1 \leq k \leq n}\left\{\frac{f\left(x_{k}\right)}{p_{k}}+\frac{f\left(\frac{\sum_{i \neq k} p_{i} x_{i}}{\sum_{i \neq k} p_{i}}\right)}{\sum_{i \neq k} p_{i}}\right\} \\
& \leq \max _{1 \leq k \leq n}\left\{\frac{f\left(x_{k}\right)}{p_{k}}+\frac{f\left(\frac{\sum_{i \neq k} p_{i} x_{i}}{\sum_{i \neq k} p_{i}}\right)}{\sum_{i \neq k} p_{i}}\right\} \\
& \leq \sum_{i=1}^{n} \frac{f\left(x_{i}\right)}{p_{i}} .
\end{aligned}
$$

Now we give two illustrations describing our work.

Example 2.3. Let $p_{1}, \ldots, p_{n}$ be positive numbers and $\sum_{i=1}^{n} p_{i}=1$. Consider the function $f(t)=\log t$ on $[1, \infty)$. Then $f$ is $Q$-class. Hence

$$
\log \left(\sum_{i=1}^{n} p_{i} x_{i}\right) \leq \frac{\log \left(x_{k}\right)}{p_{k}}+\frac{\log \left(\frac{\sum_{i \neq k} p_{i} x_{i}}{\sum_{i \neq k} p_{i}}\right)}{\sum_{i \neq k} p_{i}} \leq \sum_{i=1}^{n} \frac{\log \left(x_{i}\right)}{p_{i}} .
$$

Therefore

$$
\log \left(\sum_{i=1}^{n} p_{i} x_{i}\right) \leq \log \left(x_{k}^{1 / p_{k}}\left(\frac{\sum_{i \neq k} p_{i} x_{i}}{\sum_{i \neq k} p_{i}}\right)^{1 /\left(1-p_{k}\right)}\right) \leq \log \left(\prod_{i=1}^{n} x_{i}^{1 / p_{i}}\right),
$$

whence

$$
\left(\prod_{i=1}^{n} x_{i}^{p_{i}}\right) \leq\left(\sum_{i=1}^{n} p_{i} x_{i}\right) \leq\left(x_{k}^{1 / p_{k}}\left(\frac{\sum_{i \neq k} p_{i} x_{i}}{\sum_{i \neq k} p_{i}}\right)^{1 /\left(1-p_{k}\right)}\right) \leq\left(\prod_{i=1}^{n} x_{i}^{1 / p_{i}}\right) .
$$


Note that the first inequality of (2.4) is the arithmetic-geometric means inequality. Applying (2.3), we get

$$
\sum_{i=1}^{n} \frac{\log \left(x_{i}\right)}{p_{i}}-\log \left(\sum_{i=1}^{n} p_{i} x_{i}\right) \geq \frac{\log \left(x_{k}\right)}{p_{k}}+\frac{\log \left(x_{\ell}\right)}{p_{\ell}}-\frac{1}{p_{k}+p_{\ell}} \log \left(\frac{p_{k} x_{k}+p_{\ell} x_{\ell}}{p_{k}+p_{\ell}}\right) \geq 0 .
$$

Hence

$$
\frac{\prod_{i=1}^{n} x_{i}^{1 / p_{i}}}{\sum_{i=1}^{n} p_{i} x_{i}} \geq \frac{x_{k}^{1 / p_{k}} x_{\ell}^{1 / p_{\ell}}}{\left(\frac{p_{k} x_{k}+p_{\ell} x_{\ell}}{p_{k}+p_{\ell}}\right)^{1 /\left(p_{k}+p_{\ell}\right)}} \geq 1
$$

ExAmple 2.4. Let $p_{1}, \ldots, p_{n}$ be positive numbers and $\sum_{i=1}^{n} p_{i}=1$. For $f(t)=\sqrt{t}$ on $[0, \infty)$,

$$
\sum_{i=1}^{n} p_{i} \sqrt{x_{i}} \leq \sqrt{\sum_{i=1}^{n} p_{i} x_{i}} \leq \frac{\sqrt{x_{k}}}{p_{k}}+\frac{\sqrt{\left(\frac{\sum_{i \neq k} p_{i} x_{i}}{\sum_{i \neq k} p_{i}}\right)}}{\sum_{i \neq k} p_{i}} \leq \sum_{i=1}^{n} \frac{\sqrt{x_{i}}}{p_{i}}
$$

and

$$
\sum_{i=1}^{n} \frac{\sqrt{x_{i}}}{p_{i}}-\sqrt{\sum_{i=1}^{n} p_{i} x_{i}} \geq \frac{\sqrt{x_{k}}}{p_{k}}+\frac{\sqrt{x_{\ell}}}{p_{\ell}}-\frac{1}{p_{k}+p_{\ell}} \sqrt{\frac{p_{k} x_{k}+p_{\ell} x_{\ell}}{p_{k}+p_{\ell}}} \geq 0 .
$$

Note that if $n=3, p_{i}=\frac{1}{3}$ and $x_{i}=(i+r-1)^{2}(i=1,2,3)$ for some $r \in \mathbb{R}^{+}$, then all inequalities above are strict. For example, with $k=1$ we have from (2.5) that

$$
r+1<\sqrt{r^{2}+2 r+\frac{5}{3}}<3\left(r+\frac{1}{2} \sqrt{r^{2}+3 r+\frac{5}{2}}\right)<9(r+1) .
$$

Corollary 2.5. Let $f, X$ and $x_{i}(i=1, \ldots, n)$ be as above. Let $p_{i}=(1 / n)$ $(i=1, \ldots, n)$ and $J=\{1, \ldots, m\}$ for some $m<n$. Then

$$
f\left(\sum_{i=1}^{n} \frac{x_{i}}{n}\right) \leq \frac{n}{m} f\left(\frac{1}{m} \sum_{i=1}^{m} x_{i}\right)+\frac{n}{n-m} f\left(\frac{1}{n-m} \sum_{i=m+1}^{n} x_{i}\right) \leq n \sum_{i=1}^{n} f\left(x_{i}\right) .
$$

Corollary 2.6. Let $X, f, p_{i}$ and $J$ be as in Theorem 2.1. Let $y_{i} \in X$ and $x_{i}=y_{i}-$ $\sum_{j=1}^{n} p_{j} y_{j}(i=1, \ldots, n)$. Then

$$
\begin{aligned}
\sum_{i=1}^{n} \frac{1}{p_{i}} & f\left(y_{i}-\sum_{j=1}^{n} p_{j} y_{j}\right) \\
& \geq \frac{1}{P_{J}} f\left(\frac{1}{P_{J}} \sum_{i \in J} p_{i}\left(y_{i}-\sum_{j=1}^{n} p_{j} y_{j}\right)\right)+\frac{1}{\bar{P}_{J}} f\left(\frac{1}{\bar{P}_{J}} \sum_{i \in \bar{J}} p_{i}\left(y_{i}-\sum_{j=1}^{n} p_{j} y_{j}\right)\right) \\
& \geq f\left(\sum_{i \in J} p_{i}\left(y_{i}-\sum_{j=1}^{n} p_{j} y_{j}\right)+\sum_{i \in \bar{J}} p_{i}\left(y_{i}-\sum_{j=1}^{n} p_{j} y_{j}\right)\right)=f(0) .
\end{aligned}
$$


The next result is a Levinson type inequality [16] for monotone increasing functions.

THEOREM 2.7. Let $p_{1}, \ldots, p_{n}$ be positive numbers, $P=\sum_{i=1}^{n} p_{i}$ and $f$ be a nonnegative monotone increasing function on $[a, b]$. Let $x_{i}, y_{i} \in[a, b](i=1, \ldots, n)$ such that $x_{i}+y_{i}=m$ for some fixed real number $m$ and $\max _{1 \leq i \leq n} y_{i} \leq \min _{1 \leq i \leq n} x_{i}$. Then

$$
P \sum_{i=1}^{n} \frac{f\left(y_{i}\right)}{p_{i}}-f\left(\frac{1}{P} \sum_{i=1}^{n} p_{i} y_{i}\right) \leq P \sum_{i=1}^{n} \frac{f\left(x_{i}\right)}{p_{i}}-f\left(\frac{1}{P} \sum_{i=1}^{n} p_{i} x_{i}\right) .
$$

Proof. We have to show that

$$
f\left(\frac{1}{P} \sum_{i=1}^{n} p_{i} x_{i}\right)-f\left(\frac{1}{P} \sum_{i=1}^{n} p_{i} y_{i}\right) \leq P \sum_{i=1}^{n} \frac{f\left(x_{i}\right)}{p_{i}}-P \sum_{i=1}^{n} \frac{f\left(y_{i}\right)}{p_{i}}
$$

or equivalently

$$
f\left(\frac{1}{P} \sum_{i=1}^{n} p_{i} x_{i}\right)-f\left(m-\frac{1}{P} \sum_{i=1}^{n} p_{i} x_{i}\right) \leq P \sum_{i=1}^{n} \frac{f\left(x_{i}\right)}{p_{i}}-P \sum_{i=1}^{n} \frac{f\left(m-x_{i}\right)}{p_{i}}
$$

To this end, we apply (1.2) to the $Q$-class function $g(x)=f(x)-f(m-x)$.

\section{Jointly $Q$-class functions}

In this section, we define a jointly $Q$-class function, analogous to joint convexity.

Definition 3.1. A function $f:[a, b] \times[c, d] \rightarrow \mathbb{R}$ is said to be jointly $Q$-class if

$$
f\left(\lambda(x, y)+(1-\lambda)\left(x^{\prime}, y^{\prime}\right)\right) \leq \frac{f(x, y)}{\lambda}+\frac{f\left(x^{\prime}, y^{\prime}\right)}{1-\lambda}
$$

for all $x, x^{\prime} \in[a, b], y, y^{\prime} \in[c, d]$ and all $\lambda \in(0,1)$.

Let $f:[0, \infty) \rightarrow \mathbb{R}$ be a $Q$-class function. Let $x=\left(x_{1}, \ldots, x_{n}\right)$ and $y=\left(y_{1}, \ldots, y_{n}\right)$ be $n$-tuples of positive numbers. Set

$$
\Delta_{f}(x, y):=\sum_{i=1}^{n} \frac{1}{x_{i}} f\left(\frac{y_{i}}{x_{i}}\right) .
$$

We have the following theorem.

Theorem 3.2. Let $f:[0, \infty) \rightarrow[0, \infty)$ be a function and $x=\left(x_{1}, \ldots, x_{n}\right)$ and $y=\left(y_{1}, \ldots, y_{n}\right)$ be n-tuples of positive numbers. Then:

(i) if $f$ is $Q$-class, then $\Delta_{f}(x, y)$ is jointly $Q$-class in $x$ and $y$;

(ii) $0 \leq(1 / X) f\left(\sum_{i=1}^{n} y_{i} / \sum_{i=1}^{n} x_{i}\right) \leq \Delta_{f}(x, y)$, for every $x, y \in(0, \infty)$, where $X=$ $\sum_{i=1}^{n} x_{i}$. 
Proof. (i) Let $\lambda \in(0,1)$ and let $x=\left(x_{1}, \ldots, x_{n}\right), x^{\prime}=\left(x_{1}^{\prime}, \ldots, x_{n}^{\prime}\right), y=\left(y_{1}, \ldots, y_{n}\right)$ and $y^{\prime}=\left(y_{1}^{\prime}, \ldots, y_{n}^{\prime}\right)$ be $n$-tuples of positive numbers. Then

$$
\begin{aligned}
\Delta_{f}\left(\lambda x+(1-\lambda) x^{\prime}, \lambda y+(1-\lambda) y^{\prime}\right) & =\sum_{i=1}^{n} \frac{1}{\lambda x_{i}+(1-\lambda) x_{i}^{\prime}} f\left(\frac{\lambda y_{i}+(1-\lambda) y_{i}^{\prime}}{\lambda x_{i}+(1-\lambda) x_{i}^{\prime}}\right) \\
& =\sum_{i=1}^{n} \frac{1}{\lambda x_{i}+(1-\lambda) x_{i}^{\prime}} f\left(\frac{\frac{\lambda}{x_{i}^{\prime}} \frac{y_{i}}{x_{i}}+\frac{1-\lambda}{x_{i}} \frac{y_{i}^{\prime}}{x_{i}^{\prime}}}{\frac{\lambda}{x_{i}^{\prime}}+\frac{1-\lambda}{x_{i}}}\right) \\
& =\sum_{i=1}^{n} \frac{1}{\lambda x_{i}+(1-\lambda) x_{i}^{\prime}} f\left(\mu \frac{y_{i}}{x_{i}}+(1-\mu) \frac{y_{i}^{\prime}}{x_{i}^{\prime}}\right),
\end{aligned}
$$

where

$$
\mu=\frac{\frac{\lambda}{x_{i}^{\prime}}}{\frac{\lambda}{x_{i}^{\prime}}+\frac{1-\lambda}{x_{i}}}=\frac{\lambda x_{i}}{\lambda x_{i}+(1-\lambda) x_{i}^{\prime}} .
$$

Since $f$ is $Q$-class,

$$
\begin{aligned}
\sum_{i=1}^{n} & \frac{1}{\lambda x_{i}+(1-\lambda) x_{i}^{\prime}} f\left(\mu \frac{y_{i}}{x_{i}}+(1-\mu) \frac{y_{i}^{\prime}}{x_{i}^{\prime}}\right) \\
& \leq \sum_{i=1}^{n} \frac{1}{\lambda x_{i}+(1-\lambda) x_{i}^{\prime}}\left(\frac{1}{\mu} f\left(\frac{y_{i}}{x_{i}}\right)+\frac{1}{1-\mu} f\left(\frac{y_{i}^{\prime}}{x_{i}^{\prime}}\right)\right) \\
& =\sum_{i=1}^{n} \frac{1}{\lambda x_{i}+(1-\lambda) x_{i}^{\prime}}\left(\frac{\lambda x_{i}+(1-\lambda) x_{i}^{\prime}}{\lambda x_{i}} f\left(\frac{y_{i}}{x_{i}}\right)+\frac{\lambda x_{i}+(1-\lambda) x_{i}^{\prime}}{(1-\lambda) x_{i}^{\prime}} f\left(\frac{y_{i}^{\prime}}{x_{i}^{\prime}}\right)\right) \\
& =\frac{1}{\lambda} \sum_{i=1}^{n} \frac{1}{x_{i}} f\left(\frac{x_{i}}{y_{i}}\right)+\frac{1}{1-\lambda} \sum_{i=1}^{n} \frac{1}{x_{i}^{\prime}} f\left(\frac{x_{i}^{\prime}}{y_{i}^{\prime}}\right)=\frac{1}{\lambda} \Delta_{f}(x, y)+\frac{1}{1-\lambda} \Delta_{f}\left(x^{\prime}, y^{\prime}\right) .
\end{aligned}
$$

(ii) Note that every $Q$-class function is nonnegative [11], so the first inequality is valid. For the second inequality,

$$
f\left(\frac{\sum_{i=1}^{n} y_{i}}{\sum_{i=1}^{n} x_{i}}\right)=f\left(\frac{\sum_{i=1}^{n} x_{i} \frac{y_{i}}{x_{i}}}{\sum_{i=1}^{n} x_{i}}\right) \leq \sum_{i=1}^{n} \frac{\sum_{k=1}^{n} x_{k}}{x_{i}} f\left(\frac{y_{i}}{x_{i}}\right)=X \Delta_{f}(x, y) .
$$

This concludes the proof.

Corollary 3.3. Let $f$ be a $Q$-class function. Let $x_{i j}, y_{i j}, p_{j}(1 \leq i \leq m, 1 \leq j \leq n)$ be positive numbers such that $\sum_{j=1}^{n} p_{j}=1$. Then

$$
\sum_{i=1}^{m} \frac{1}{\sum_{j=1}^{n} p_{j} x_{i j}} f\left(\frac{\sum_{j=1}^{n} p_{j} y_{i j}}{\sum_{j=1}^{n} p_{j} x_{i j}}\right)
$$




$$
\begin{aligned}
& \leq P_{J} \sum_{i=1}^{m} \frac{1}{\sum_{j \in J} p_{j} x_{i j}} f\left(\frac{\sum_{j \in J} p_{j} y_{i j}}{\sum_{j \in J} p_{j} x_{i j}}\right)+\bar{P}_{J} \sum_{i=1}^{m} \frac{1}{\sum_{j \in \bar{J}} p_{j} x_{i j}} f\left(\frac{\sum_{j \in \bar{J}} p_{j} y_{i j}}{\sum_{j \in \bar{J}} p_{j} x_{i j}}\right) \\
& \leq \sum_{j=1}^{n} \sum_{i=1}^{m} \frac{1}{x_{i j} p_{j}} f\left(\frac{y_{i j}}{x_{i j}}\right) .
\end{aligned}
$$

Proof. This follows from Theorems 2.1 and 3.2.

The next theorem gives a lower bound for the function $\Delta_{f}$.

Theorem 3.4. Let $f$ be a $Q$-class function. Let $x=\left(x_{1}, \ldots, x_{n}\right)$ and $p=\left(p_{1}, \ldots, p_{n}\right)$ be n-tuples of positive numbers such that $\sum_{i=1}^{n} p_{i}=1$. Let $J$ be a nonempty proper subset of $\{1, \ldots, n\}$ and $\bar{J}=\{1, \ldots, n\}-J$. Put $X_{J}=\sum_{i \in J} x_{i}$ and $P_{J}=\sum_{i \in J} p_{i}$. Then

$$
0 \leq f\left(\sum_{i=1}^{n} x_{i}\right) \leq \frac{1}{P_{J}} f\left(\frac{X_{J}}{P_{J}}\right)+\frac{1}{1-P_{J}} f\left(\frac{\sum_{i \in \bar{J}} x_{i}}{1-P_{J}}\right) \leq \Delta_{f}(p, x) .
$$

Proof. The left inequality follows from Theorem 2.1. The proof of the right inequality is similar to that of Theorem 3.2 and we omit it.

ExAmple 3.5. Let $f(t)=\log t$, and $x_{1}, \ldots, x_{n}$ and $p_{1}, \ldots, p_{n}$ be positive numbers such that $x_{i} \geq p_{i}$ and $\sum_{i=1}^{n} p_{i}=1$. Let $J=\{k\}$ for some $k \in\{1, \ldots, n\}$. It follows from Theorem 3.4 that

$$
0 \leq \log \left(\sum_{i=1}^{n} x_{i}\right) \leq \frac{1}{p_{k}} \log \left(\frac{x_{k}}{p_{k}}\right)+\frac{1}{1-p_{k}} \log \left(\frac{\sum_{i \neq k} x_{i}}{1-p_{k}}\right) \leq \sum_{i=1}^{n} \frac{1}{p_{i}} \log \left(\frac{x_{i}}{p_{i}}\right) .
$$

Therefore, it follows from the monotonicity of the function $e^{t}$ that

$$
1 \leq \sum_{i=1}^{n} x_{i} \leq \sqrt[p_{k}]{\frac{x_{k}}{p_{k}}} \sqrt[1-p_{k}]{\frac{\sum_{i \neq k} x_{i}}{1-p_{k}}} \leq \prod_{i=1}^{n} \sqrt[p_{i}]{\frac{x_{i}}{p_{i}}}
$$

The next theorem gives a Jensen type inequality for jointly $Q$-class functions.

Theorem 3.6. Let $f: I \times J \rightarrow \mathbb{R}$ be a jointly $Q$-class function. Let $x_{1}, \ldots, x_{n} \in I$ and $y_{1}, \ldots, y_{n} \in J$. Let $p_{i}, q_{i}>0(i=1, \ldots, n), P=\sum_{i=1}^{n} p_{i}$ and $Q=\sum_{i=1}^{n} q_{i}$. Put

$$
\bar{x}=\frac{1}{P} \sum_{i=1}^{n} p_{i} x_{i} \quad \text { and } \quad \bar{y}=\frac{1}{Q} \sum_{i=1}^{n} q_{i} y_{i} .
$$

Then

$$
f(\bar{x}, \bar{y}) \leq \frac{1}{2}\left(P \sum_{i=1}^{n} \frac{f\left(x_{i}, \bar{y}\right)}{p_{i}}+Q \sum_{j=1}^{n} \frac{f\left(\bar{x}, y_{j}\right)}{q_{j}}\right) \leq P Q \sum_{i=1}^{n} \sum_{j=1}^{n} \frac{f\left(x_{i}, y_{j}\right)}{p_{i} q_{j}} .
$$


Proof. Since $f$ is jointly $Q$-class, it is clearly $Q$-class in each of its variables, from which we get

$$
\begin{aligned}
& f\left(x_{i}, \bar{y}\right) \leq Q \sum_{j=1}^{n} \frac{f\left(x_{i}, y_{j}\right)}{q_{j}} \quad(i=1, \ldots, n), \\
& f\left(\bar{x}, y_{j}\right) \leq P \sum_{i=1}^{n} \frac{f\left(x_{i}, y_{j}\right)}{p_{i}} \quad(j=1, \ldots, n) .
\end{aligned}
$$

Multiplying both sides of (3.2) and (3.3) by $1 / p_{i}$ and $1 / q_{j}$, respectively, and summing over $i$ and $j$, we obtain

$$
\begin{aligned}
\sum_{i=1}^{n} \frac{f\left(x_{i}, \bar{y}\right)}{p_{i}} \leq Q \sum_{i=1}^{n} \sum_{j=1}^{n} \frac{f\left(x_{i}, y_{j}\right)}{p_{i} q_{j}} \\
\sum_{j=1}^{n} \frac{f\left(\bar{x}, y_{j}\right)}{q_{j}} \leq P \sum_{i=1}^{n} \sum_{j=1}^{n} \frac{f\left(x_{i}, y_{j}\right)}{p_{i} q_{j}} .
\end{aligned}
$$

Multiplying (3.4) and (3.5), respectively, by $P$ and $Q$ and adding both inequalities, we get the second inequality in (3.1). To get the first one, note that

$$
\begin{aligned}
& f(\bar{x}, \bar{y})=f\left(\frac{1}{P} \sum_{i=1}^{n} p_{i} x_{i}, \bar{y}\right) \leq P \sum_{i=1}^{n} \frac{f\left(x_{i}, \bar{y}\right)}{p_{i}} \\
& f(\bar{x}, \bar{y})=f\left(\bar{x}, \frac{1}{Q} \sum_{j=1}^{n} q_{j} y_{j}\right) \leq Q \sum_{i=1}^{n} \frac{f\left(\bar{x}, y_{j}\right)}{q_{j}} .
\end{aligned}
$$

These inequalities immediately yield the first inequality of (3.1).

Let $f, P, Q, p_{i}, q_{j}, x_{i}, y_{j}, \bar{x}, \bar{y}$ be as in Theorem 3.6. Define a function $\Gamma_{f}:[0,1] \times$ $[0,1] \rightarrow \mathbb{R}$ by

$$
\Gamma_{f}(t, s):=P Q \sum_{i=1}^{n} \sum_{j=1}^{n} \frac{f\left(t x_{i}+(1-t) \bar{x}, s y_{j}+(1-s) \bar{y}\right)}{p_{i} q_{j}}
$$

\section{THEOREM 3.7. With notation as above:}

(i) $\Gamma_{f}$ is jointly $Q$-class;

(ii) $0 \leq f(\bar{x}, \bar{y}) \leq \Gamma_{f}(t, s)$ for each $t, s \in[0,1]$.

Proof. (i) Let $\lambda \in(0,1)$ and $t_{1}, t_{2}, s_{1}, s_{2} \in[0,1]$. Then

$$
\begin{aligned}
\Gamma_{f}\left(\lambda t_{1}+\right. & \left.(1-\lambda) t_{2}, \lambda s_{1}+(1-\lambda) s_{2}\right) \\
= & P Q \sum_{i=1}^{n} \sum_{j=1}^{n} \frac{1}{p_{i} q_{j}} f\left(\left(\lambda t_{1}+(1-\lambda) t_{2}\right) x_{i}+\left(1-\left(\lambda t_{1}+(1-\lambda) t_{2}\right)\right) \bar{x}\right. \\
& \left.\left(\lambda s_{1}+(1-\lambda) s_{2}\right) y_{j}+\left(1-\left(\lambda s_{1}+(1-\lambda) s_{2}\right)\right) \bar{y}\right) \\
= & P Q \sum_{i=1}^{n} \sum_{j=1}^{n} \frac{1}{p_{i} q_{j}} f\left(\lambda\left(t_{1} x_{i}+\left(1-t_{1}\right) \bar{x}, s_{1} y_{j}+\left(1-s_{1}\right) \bar{y}\right)\right.
\end{aligned}
$$




$$
\begin{aligned}
& \left.+(1-\lambda)\left(t_{2} x_{i}+\left(1-t_{2}\right) \bar{x}, s_{2} y_{j}+\left(1-s_{2}\right) \bar{y}\right)\right) \\
\leq & \frac{P Q}{\lambda} \sum_{i=1}^{n} \sum_{j=1}^{n} \frac{f\left(\left(t_{1} x_{i}+\left(1-t_{1}\right) \bar{x}, s_{1} y_{j}+\left(1-s_{1}\right) \bar{y}\right)\right)}{p_{i} q_{j}} \\
& +\frac{P Q}{1-\lambda} \sum_{i=1}^{n} \sum_{j=1}^{n} \frac{f\left(\left(t_{2} x_{i}+\left(1-t_{2}\right) \bar{x}, s_{2} y_{j}+\left(1-s_{2}\right) \bar{y}\right)\right)}{p_{i} q_{j}} \\
= & \frac{1}{\lambda} \Gamma_{f}\left(t_{1}, s_{1}\right)+\frac{1}{1-\lambda} \Gamma_{f}\left(t_{2}, s_{2}\right) .
\end{aligned}
$$

(ii) Using the Jensen inequality for the $Q$-class function $f$, we get

$$
\begin{aligned}
\Gamma_{f}(t, s) & =P Q \sum_{i=1}^{n} \sum_{j=1}^{n} \frac{f\left(t x_{i}+(1-t) \bar{x}, s y_{j}+(1-s) \bar{y}\right)}{p_{i} q_{j}} \\
& =P \sum_{i=1}^{n} \frac{Q}{p_{i}} \sum_{j=1}^{n} \frac{f\left(t x_{i}+(1-t) \bar{x}, s y_{j}+(1-s) \bar{y}\right)}{q_{j}} \\
& \geq P \sum_{i=1}^{n} \frac{1}{p_{i}} f\left(t x_{i}+(1-t) \bar{x}, \frac{1}{Q} \sum_{j=1}^{n} q_{j}\left(s y_{j}+(1-s) \bar{y}\right)\right) \\
& \geq f\left(\frac{t}{P} \sum_{i=1}^{n} p_{i} x_{i}+(1-t) \bar{x}, \frac{s}{Q} \sum_{j=1}^{n} q_{j} y_{j}+(1-s) \bar{y}\right) \\
& =f(\bar{x}, \bar{y}) .
\end{aligned}
$$

This concludes the proof.

\section{Ostrowski and Hermite-Hadamard type inequalities}

We now turn our attention to the Ostrowski and Hermite-Hadamard inequalities. First, we state some Hermite-Hadamard inequalities related to $Q$-class functions. We need the following lemma for our next result.

Lemma 4.1 [2]. Let $f: I \subseteq \mathbb{R} \rightarrow \mathbb{R}$ be a twice differential mapping on $I^{\circ}, a, b \in I$ with $a<b$ and $f^{\prime \prime}$ integrable on $[a, b]$. Then

$$
\frac{f(a)+f(b)}{2}-\frac{1}{b-a} \int_{a}^{b} f(x) d x=\frac{(b-a)^{2}}{2} \int_{0}^{1} t(1-t) f^{\prime \prime}(t a+(1-t) b) d t .
$$

Theorem 4.2. Let $f$ be a function as in Lemma 4.1. If $\left|f^{\prime \prime}\right|$ is $Q$-class, then

$$
\left|\frac{f(a)+f(b)}{2}-\frac{1}{b-a} \int_{a}^{b} f(x) d x\right| \leq \frac{(b-a)^{2}}{4}\left(\left|f^{\prime \prime}(a)\right|+\left|f^{\prime \prime}(b)\right|\right) .
$$


Proof. It follows from Lemma 4.1 that

$$
\begin{aligned}
\left|\frac{f(a)+f(b)}{2}-\frac{1}{b-a} \int_{a}^{b} f(x) d x\right| & \leq \frac{(b-a)^{2}}{2} \int_{0}^{1} t(1-t)\left|f^{\prime \prime}(t a+(1-t) b)\right| d t \\
& \leq \frac{(b-a)^{2}}{2} \int_{0}^{1} t(1-t)\left(\frac{f^{\prime \prime}(a)}{t}+\frac{f^{\prime \prime}(b)}{1-t}\right) d t \\
& =\frac{(b-a)^{2}}{2}\left(\int_{0}^{1}(1-t)\left|f^{\prime \prime}(a)\right| d t+\int_{0}^{1} t\left|f^{\prime \prime}(b)\right| d t\right) \\
& =\frac{(b-a)^{2}}{4}\left(\left|f^{\prime \prime}(a)\right|+\left|f^{\prime \prime}(b)\right|\right) .
\end{aligned}
$$

This concludes the proof.

THEOREM 4.3. Let $f$ be a function as in Lemma 4.1. If $\left|f^{\prime \prime}\right|^{q}$ is $Q$-class for some $q>1$, then

$$
\begin{aligned}
& \left|\frac{f(a)+f(b)}{2}-\frac{1}{b-a} \int_{a}^{b} f(x) d x\right| \\
& \quad \leq(b-a)^{2}\left(\frac{1}{2}\right)^{1+(1 / q)}\left(\frac{1}{6}\right)^{1-(1 / q)}\left(\left|f^{\prime \prime}(a)\right|^{q}+\left|f^{\prime \prime}(b)\right|^{q}\right)^{1 / q} .
\end{aligned}
$$

Proof. It follows from Lemma 4.1 that

$$
\begin{aligned}
\mid \frac{f(a)}{2}+f(b) & \frac{1}{b-a} \int_{a}^{b} f(x) d x \mid \\
& \leq \frac{(b-a)^{2}}{2} \int_{0}^{1} t(1-t)\left|f^{\prime \prime}(t a+(1-t) b)\right| d t \\
& \leq \frac{(b-a)^{2}}{2}\left(\int_{0}^{1} t(1-t) d t\right)^{1-(1 / q)}\left(\int_{0}^{1} t(1-t)\left|f^{\prime \prime}(t a+(1-t) b)\right|^{q} d t\right)^{1 / q} \\
& \leq \frac{(b-a)^{2}}{2}\left(\frac{1}{6}\right)^{1-(1 / q)}\left(\left|f^{\prime \prime}(a)\right|^{q} \int_{0}^{1}(1-t) d t+\left|f^{\prime \prime}(b)\right|^{q} \int_{0}^{1} t d t\right)^{1 / q} \\
& \leq \frac{(b-a)^{2}}{2}\left(\frac{1}{6}\right)^{1-(1 / q)}\left(\frac{\left|f^{\prime \prime}(a)\right|^{q}+\left|f^{\prime \prime}(b)\right|^{q}}{2}\right)^{1 / q} \\
& =(b-a)^{2}\left(\frac{1}{2}\right)^{1+(1 / q)}\left(\frac{1}{6}\right)^{1-(1 / q)}\left(\left|f^{\prime \prime}(a)\right|^{q}+\left|f^{\prime \prime}(b)\right|^{q}\right)^{1 / q} .
\end{aligned}
$$

This concludes the proof.

To prove the next two theorems, which give some Ostrowski type inequalities, we need the following lemma. 
Lemma 4.4 [1]. Let $f: I \subseteq \mathbb{R} \rightarrow \mathbb{R}$ be a differential mapping on $I^{\circ}, a, b \in I$ with $a<b$ and $f^{\prime}$ integrable on $[a, b]$. Then

$$
\begin{aligned}
f(x) & -\frac{1}{b-a} \int_{a}^{b} f(u) d u \\
& =(b-a)\left(\int_{0}^{m} t f^{\prime}(t a+(1-t) b) d t+\int_{m}^{1}(1-t) f^{\prime}(t a+(1-t) b) d t\right),
\end{aligned}
$$

where $m=(b-x) /(b-a)$.

THeOREM 4.5. Let $f$ be a function as in Lemma 4.4. If $\left|f^{\prime}\right|$ is $Q$-class, then

$$
\begin{aligned}
\mid f(x) & -\frac{1}{b-a} \int_{a}^{b} f(u) d u \mid \\
& \leq(b-a)\left(\left|f^{\prime}(a)\right|\left(\log \left|\frac{b-a}{b-x}\right|+\frac{b+a-2 x}{b-a}\right)+\left|f^{\prime}(b)\right|\left(\log \left|\frac{b-a}{x-a}\right|+\frac{2 x-a-b}{b-a}\right)\right) .
\end{aligned}
$$

Proof. By Lemma 4.4 we have

$$
\begin{aligned}
\mid f(x)- & \frac{1}{b-a} \int_{a}^{b} f(u) d u \mid \\
\leq & (b-a)\left(\int_{0}^{m} t\left|f^{\prime}(t a+(1-t) b)\right| d t+\int_{m}^{1}(1-t)\left|f^{\prime}(t a+(1-t) b)\right| d t\right) \\
\leq & (b-a)\left(\int_{0}^{m} t\left(\frac{\left|f^{\prime}(a)\right|}{t}+\frac{\left|f^{\prime}(b)\right|}{1-t}\right) d t+\int_{m}^{1}(1-t)\left(\frac{\left|f^{\prime}(a)\right|}{t}+\frac{\left|f^{\prime}(b)\right|}{1-t}\right) d t\right) \\
= & (b-a)\left(\left|f^{\prime}(a)\right| \int_{0}^{m} d t+\left|f^{\prime}(b)\right| \int_{0}^{m} \frac{t}{1-t} d t\right. \\
& \left.+\left|f^{\prime}(a)\right| \int_{m}^{1} \frac{1-t}{t} d t+\left|f^{\prime}(b)\right| \int_{m}^{1} d t\right) \\
= & (b-a)\left(\left|f^{\prime}(a)\right|\left(\log \left|\frac{b-a}{b-x}\right|+\frac{b+a-2 x}{b-a}\right)+\left|f^{\prime}(b)\right|\left(\log \left|\frac{b-a}{x-a}\right|+\frac{2 x-a-b}{b-a}\right)\right) .
\end{aligned}
$$

This concludes the proof.

Corollary 4.6. Let $f$ and $f^{\prime}$ be as in Theorem 4.5.

$$
\left|f\left(\frac{a+b}{2}\right)-\frac{1}{b-a} \int_{a}^{b} f(u) d u\right| \leq(b-a) \log 2\left(\left|f^{\prime}(a)\right|+\left|f^{\prime}(b)\right|\right) .
$$

Proof. Put $x=(a+b) / 2$ in Theorem 4.5. 
Theorem 4.7. Let $f$ be a function as in Lemma 4.4. If $\left|f^{\prime}\right|^{q}$ is $Q$-class for some $q \geq 1$, then

$$
\begin{aligned}
\mid f(x)- & \frac{1}{b-a} \int_{a}^{b} f(u) d u \mid \\
\leq & (b-a)\left(\frac{1}{2}\left(\frac{b-x}{b-a}\right)^{2(1-(1 / q))}\left(\left|f^{\prime}(a)\right|^{q} \frac{b-x}{b-a}+\left|f^{\prime}(b)\right|^{q}\left(\log \left|\frac{b-a}{x-a}\right|-\frac{b-x}{b-a}\right)\right)^{1 / q}\right) \\
& +(b-a)\left(\frac{2 x-a-b}{2 b-2 a}+\frac{1}{2}\left(\frac{b-x}{b-a}\right)^{2}\right)^{1-(1 / q)} \\
& \times\left(\left|f^{\prime}(b)\right|^{q} \frac{x-a}{b-a}+\left|f^{\prime}(a)\right|^{q}\left(\log \left|\frac{b-a}{b-x}\right|-\frac{x-a}{b-a}\right)\right)^{1 / q} .
\end{aligned}
$$

Proof. It follows from Lemma 4.4 that

$$
\begin{aligned}
\mid f(x) & -\frac{1}{b-a} \int_{a}^{b} f(u) d u \mid \\
\leq & (b-a)\left(\int_{0}^{m} t\left|f^{\prime}(t a+(1-t) b)\right| d t+\int_{m}^{1}(1-t)\left|f^{\prime}(t a+(1-t) b)\right| d t\right) \\
\leq & (b-a)\left(\left(\int_{0}^{m} t d t\right)^{1-(1 / q)}\left(\int_{0}^{m} t\left|f^{\prime}(t a+(1-t) b)\right|^{q} d t\right)^{1 / q}\right. \\
& \left.+\left(\int_{m}^{1}(1-t) d t\right)^{1-(1 / q)}\left(\int_{m}^{1}(1-t)\left|f^{\prime}(t a+(1-t) b)\right|^{q} d t\right)^{1 / q}\right),
\end{aligned}
$$

by the power mean inequality. Since $\left|f^{\prime}\right|^{q}$ is $Q$-class,

$$
\begin{aligned}
\int_{0}^{m} t\left|f^{\prime}(t a+(1-t) b)\right|^{q} d t & \leq \int_{0}^{m} t\left(\frac{\left|f^{\prime}(a)\right|^{q}}{t}+\frac{\left|f^{\prime}(b)\right|^{q}}{1-t}\right) d t \\
& =\left|f^{\prime}(a)\right|^{q} \int_{0}^{m} d t+\left|f^{\prime}(b)\right|^{q} \int_{0}^{m} \frac{t}{1-t} d t \\
& =\left|f^{\prime}(a)\right|^{q} \frac{b-x}{b-a}+\left|f^{\prime}(b)\right|^{q}\left(\log \left|\frac{b-a}{x-a}\right|-\frac{b-x}{b-a}\right)
\end{aligned}
$$

and

$$
\begin{aligned}
\int_{m}^{1}(1-t)\left|f^{\prime}(t a+(1-t) b)\right|^{q} d t & \leq \int_{m}^{1}(1-t)\left(\frac{\left|f^{\prime}(a)\right|^{q}}{t}+\frac{\left|f^{\prime}(b)\right|^{q}}{1-t}\right) d t \\
& =\left|f^{\prime}(a)\right|^{q} \int_{m}^{1} \frac{1-t}{t} d t+\left|f^{\prime}(b)\right|^{q} \int_{m}^{1} d t \\
& =\left|f^{\prime}(b)\right|^{q} \frac{x-a}{b-a}+\left|f^{\prime}(a)\right|^{q}\left(\log \left|\frac{b-a}{b-x}\right|-\frac{x-a}{b-a}\right) .
\end{aligned}
$$


Hence

$$
\begin{aligned}
\mid f(x)- & \frac{1}{b-a} \int_{a}^{b} f(u) d u \mid \\
\leq & (b-a)\left(\frac{1}{2}\left(\frac{b-x}{b-a}\right)^{2(1-(1 / q))}\left(\left|f^{\prime}(a)\right|^{q} \frac{b-x}{b-a}+\left|f^{\prime}(b)\right|^{q}\left(\log \left|\frac{b-a}{x-a}\right|-\frac{b-x}{b-a}\right)\right)^{1 / q}\right) \\
& +(b-a)\left(\frac{2 x-a-b}{2 b-2 a}+\frac{1}{2}\left(\frac{b-x}{b-a}\right)^{2}\right)^{1-(1 / q)} \\
& \times\left(\left|f^{\prime}(b)\right|^{q} \frac{x-a}{b-a}+\left|f^{\prime}(a)\right|^{q}\left(\log \left|\frac{b-a}{b-x}\right|-\frac{x-a}{b-a}\right)\right)^{1 / q} .
\end{aligned}
$$

This concludes the proof.

\section{References}

[1] M. Alomari and M. Darus, 'Some Ostrowski type inequalities for convex functions with applications', RGMIA 13(1) (2010), No. 3. Article 3.

[2] M. Alomari, M. Darus and S. S. Dragomir, 'New inequalities of Hermite-Hadamard type for functions whose second derivatives absolute values are quasiconvex', RGMIA Res. Rep. Coll. 12 (2009), Supplement, Article 14, online: http://www.staff.vu.edu.au/RGMIA/v12(E).asp.

[3] M. Alomari, M. Darus, S. S. Dragomir and P. Cerone, 'Ostrowski type inequalities for functions whose derivatives are s-convex in the second sense', Appl. Math. Lett. 23(9) (2010), 1071-1076.

[4] S. Barza, J. Pecaric and L.-E. Persson, 'Reversed Hölder type inequalities for monotone functions of several variables', Math. Nachr. 186 (1997), 67-80.

[5] A. Čivljak and Lj. Dedić, 'Generalizations of Ostrowski inequality via biparametric Euler harmonic identities for measures', Banach J. Math. Anal. 4(1) (2010), 170-184.

[6] S. S. Dragomir, 'Superadditivity of some functionals associated with Jensen's inequality for convex functions on linear spaces with applications', Bull. Aust. Math. Soc. 82(1) (2010), 44-61.

[7] S. S. Dragomir and R. P. Agarwal, 'Two inequalities for differentiable mappings and applications to special means of real numbers and to trapezoidal formula', Appl. Math. Lett. 11 (1998), 91-95.

[8] S. S. Dragomir and C. E. M. Pearce, 'On Jensen's inequality for a class of functions of Godunova and Levin', Period. Math. Hungar. 33(2) (1996), 93-100.

[9] S. S. Dragomir, J. Pečarić and L.-E. Persson, 'Some inequalities of Hadamard type', Soochow J. Math. 21(3) (1995), 335-341.

[10] M. Emin Özdemir, M. Avci and E. Set, 'On some inequalities of Hermite-Hadamard type via m-convexity', Appl. Math. Lett. 23(9) (2010), 1065-1070.

[11] J. I. Fujii, M. Kian and M. S. Moslehian, 'Operator Q-class functions', Sci. Math. Jpn. 73(1) (2011), 75-80.

[12] E. K. Godunova and V. I. Levin, 'Inequalities for functions of a broad class that contains convex, monotone and some other forms of functions', in: Numerical Mathematics and Mathematical Physics (Moskov. Gos. Ped. Inst, Moscow, 1985), pp. 138-142, 166 (in Russian).

[13] E. Kikianty, S. S. Dragomir and P. Cerone, 'Sharp inequalities of Ostrowski type for convex functions defined on linear spaces and application', Comput. Math. Appl. 56(9) (2008), 2235-2246.

[14] B. M. Klaričić Bakula and J. Pečarić, 'On the Jensen's inequality for convex functions on the co-ordinates in a rectangle from the plane', Taiwanese J. Math. 10(5) (2006), 1271-1292.

[15] D. Mitrinović and J. Pečarić, 'Note on a class of functions of Godunova and Levin', C. R. Math. Rep. Acad. Sci. Can. 12 (1990), 33-36. 
[16] J. Pečarić, F. Proschan and Y. L. Tong, Convex Functions, Partial Orderings, and Statistical Applications, Mathematics in Science and Engineering (Academic Press, Boston, 1992).

[17] M. Radulescu, S. Radulescu and P. Alexandrescu, 'On Schur inequality and Schur functions', Ann. Univ. Craiova Math. Comp. Sci. Ser. 32 (2005), 202-208.

[18] M. Radulescu, S. Radulescu and P. Alexandrescu, 'On the Godunova-Levin-Schur class of functions', Math. Inequal. Appl. 12(4) (2009), 853-862.

MOHAMMAD SAL MOSLEHIAN, Department of Pure Mathematics, Center of Excellence in Analysis on Algebraic Structures (CEAAS), Ferdowsi University of Mashhad, PO Box 1159, Mashhad 91775, Iran e-mail: moslehian@ferdowsi.um.ac.ir, moslehian@member.ams.org

MOHSEN KIAN, Department of Pure Mathematics, Center of Excellence in Analysis on Algebraic Structures (CEAAS), Ferdowsi University of Mashhad, PO Box 1159, Mashhad 91775, Iran e-mail: kian_tak@yahoo.com 